\title{
Approximating fixed points for continuous functions on an arbitrary interval
}

\section{Prasit Cholamjiak* and Nattawut Pholasa}

"Correspondence:

prasitch2008@yahoo.com

School of Science, University of

Phayao, Phayao, 56000, Thailand

\begin{abstract}
In this research article, we introduce a new iterative method for solving a fixed point problem of continuous functions on an arbitrary interval. We then prove the convergence theorem of the proposed algorithm. We finally give numerical examples to compare the result with Mann, Ishikawa and Noor iterations. Our main results extend the corresponding results in the literature.
\end{abstract}

MSC: $47 \mathrm{H} 09 ; 47 \mathrm{H} 10$

Keywords: continuous function; convergence theorem; fixed point; iteration

\section{Introduction}

Let $C$ be a closed interval on the real line and let $f: C \rightarrow C$ be a continuous function. A point $p \in C$ is called a fixed point of $f$ if $f(p)=p$.

One classical way to approximate a fixed point of a nonlinear mapping was introduced in 1953 by Mann [1] as follows: a sequence $\left\{x_{n}\right\}$ defined by $x_{1} \in C$ and

$$
x_{n+1}=\left(1-\alpha_{n}\right) x_{n}+\alpha_{n} f\left(x_{n}\right)
$$

for all $n \geq 1$, where $\left\{\alpha_{n}\right\}$ is a sequence in $[0,1]$. Such an iteration process is known as Mann iteration. In 1991, Borwein and Borwein [2] proved the convergence theorem for a continuous function on the closed and bounded interval in the real line by using iteration (1.1).

Another classical iteration process was introduced by Ishikawa [3] as follows: a sequence $\left\{x_{n}\right\}$ defined by $x_{1} \in C$ and

$$
\begin{aligned}
& y_{n}=\left(1-\beta_{n}\right) x_{n}+\beta_{n} f\left(x_{n}\right), \\
& x_{n+1}=\left(1-\alpha_{n}\right) x_{n}+\alpha_{n} f\left(y_{n}\right)
\end{aligned}
$$

for all $n \geq 1$, where $\left\{\alpha_{n}\right\}$ and $\left\{\beta_{n}\right\}$ are sequences in $[0,1]$. Such an iterative method is known as Ishikawa iteration. In 2006, Qing and Qihou [4] proved the convergence theorem of the sequence generated by iteration (1.2) for a continuous function on the closed interval in the real line (see also [5]).

0 2013 Cholamjiak and Pholasa; licensee Springer. This is an Open Access article distributed under the terms of the Creative Commons Attribution License (http://creativecommons.org/licenses/by/2.0), which permits unrestricted use, distribution, and reproduction in any medium, provided the original work is properly cited. 
In 2000, Noor [6] defined the following iterative scheme by $x_{1} \in C$ and

$$
\begin{aligned}
& z_{n}=\left(1-\mu_{n}\right) x_{n}+\mu_{n} f\left(x_{n}\right), \\
& y_{n}=\left(1-\beta_{n}\right) x_{n}+\beta_{n} f\left(z_{n}\right), \\
& x_{n+1}=\left(1-\alpha_{n}\right) x_{n}+\alpha_{n} f\left(y_{n}\right)
\end{aligned}
$$

for all $n \geq 1$, where $\left\{\alpha_{n}\right\},\left\{\beta_{n}\right\}$ and $\left\{\mu_{n}\right\}$ are sequences in $[0,1]$. Such an iterative method is known as Noor iteration. Phuengrattana and Suantai [7] considered the convergence of Noor iteration for continuous functions on an arbitrary interval in the real line.

In this paper, motivated by the previous ones, we introduce a new modified iteration process for solving a fixed point problem for continuous functions on an arbitrary interval in the real line. Numerical examples are also presented to compare the result with Mann, Ishikawa and Noor iterations.

\section{Main results}

We begin this section by proving the following crucial lemmas.

Lemma 2.1 Let $C$ be a closed interval on the real line (can be unbounded) and let $f: C \rightarrow C$ be a continuous function. Let $\left\{\alpha_{n}\right\},\left\{\beta_{n}\right\},\left\{\mu_{n}\right\},\left\{\gamma_{n}\right\}$ and $\left\{\tau_{n}\right\}$ be sequences in $[0,1]$ with $0 \leq \tau_{n}+\beta_{n} \leq 1$ and $0 \leq \gamma_{n}+\alpha_{n} \leq 1$. Let $\left\{x_{n}\right\}$ be a sequence generated iteratively by $x_{1} \in C$ and

$$
\begin{aligned}
& z_{n}=\left(1-\mu_{n}\right) x_{n}+\mu_{n} f\left(x_{n}\right), \\
& y_{n}=\left(1-\tau_{n}-\beta_{n}\right) x_{n}+\tau_{n} z_{n}+\beta_{n} f\left(z_{n}\right), \\
& x_{n+1}=\left(1-\gamma_{n}-\alpha_{n}\right) z_{n}+\gamma_{n} y_{n}+\alpha_{n} f\left(y_{n}\right), \quad n \geq 1,
\end{aligned}
$$

where $\sum_{n=1}^{\infty} \alpha_{n}=\infty, \lim _{n \rightarrow \infty} \alpha_{n}=0, \sum_{n=1}^{\infty} \beta_{n}<\infty$ and $\sum_{n=1}^{\infty} \mu_{n}<\infty$.

If $x_{n} \rightarrow a$, then $a$ is a fixed point off.

Proof Let $x_{n} \rightarrow a$ and suppose $a \neq f(a)$. Then $\left\{x_{n}\right\}$ is bounded. So, $\left\{f\left(x_{n}\right)\right\}$ is bounded by the continuity of $f$. So are $\left\{y_{n}\right\},\left\{z_{n}\right\},\left\{f\left(y_{n}\right)\right\}$ and $\left\{f\left(z_{n}\right)\right\}$. Moreover, $z_{n} \rightarrow a$ since $x_{n} \rightarrow a$ and $\mu_{n} \rightarrow 0$. We also have $y_{n} \rightarrow a$ since $z_{n} \rightarrow a$ and $\beta_{n} \rightarrow 0$.

From (2.1) we obtain

$$
\begin{aligned}
x_{n+1}= & \left(1-\gamma_{n}-\alpha_{n}\right) z_{n}+\gamma_{n} y_{n}+\alpha_{n} f\left(y_{n}\right) \\
= & z_{n}+\gamma_{n}\left(y_{n}-z_{n}\right)+\alpha_{n}\left(f\left(y_{n}\right)-z_{n}\right) \\
= & \left(1-\mu_{n}\right) x_{n}+\mu_{n} f\left(x_{n}\right)+\gamma_{n}\left(y_{n}-z_{n}\right)+\alpha_{n}\left(f\left(y_{n}\right)-z_{n}\right) \\
= & x_{n}+\mu_{n}\left(f\left(x_{n}\right)-x_{n}\right)+\gamma_{n}\left(y_{n}-z_{n}\right)+\alpha_{n}\left(f\left(y_{n}\right)-z_{n}\right) \\
= & x_{n}+\mu_{n}\left(f\left(x_{n}\right)-x_{n}\right)+\gamma_{n}\left(\left(1-\tau_{n}-\beta_{n}\right)\left(x_{n}-z_{n}\right)+\beta_{n}\left(f\left(z_{n}\right)-z_{n}\right)\right) \\
& +\alpha_{n}\left(f\left(y_{n}\right)-z_{n}\right) \\
= & x_{n}+\mu_{n}\left(f\left(x_{n}\right)-x_{n}\right)+\gamma_{n}\left(\left(1-\tau_{n}-\beta_{n}\right) \mu_{n}\left(x_{n}-f\left(x_{n}\right)\right)+\beta_{n}\left(f\left(z_{n}\right)-z_{n}\right)\right) \\
& +\alpha_{n}\left(f\left(y_{n}\right)-z_{n}\right)
\end{aligned}
$$




$$
\begin{aligned}
= & x_{n}+\mu_{n}\left(1-\gamma_{n}\left(1-\tau_{n}-\beta_{n}\right)\right)\left(f\left(x_{n}\right)-x_{n}\right)+\gamma_{n} \beta_{n}\left(f\left(z_{n}\right)-z_{n}\right) \\
& +\alpha_{n}\left(f\left(y_{n}\right)-z_{n}\right) .
\end{aligned}
$$

Let $p_{k}=f\left(x_{k}\right)-x_{k}, q_{k}=f\left(z_{k}\right)-z_{k}$ and $r_{k}=f\left(y_{k}\right)-z_{k}$. Then we observe

$$
\begin{aligned}
& \lim _{k \rightarrow \infty} p_{k}=\lim _{n \rightarrow \infty}\left(f\left(x_{k}\right)-x_{k}\right)=f(a)-a \neq 0, \\
& \lim _{k \rightarrow \infty} q_{k}=\lim _{n \rightarrow \infty}\left(f\left(z_{k}\right)-z_{k}\right)=f(a)-a \neq 0, \\
& \lim _{k \rightarrow \infty} r_{k}=\lim _{n \rightarrow \infty}\left(f\left(y_{k}\right)-z_{k}\right)=f(a)-a \neq 0 .
\end{aligned}
$$

So, from (2.2) we obtain

$$
\begin{aligned}
x_{n}= & x_{1}+\sum_{k=1}^{n} \mu_{k}\left(1-\gamma_{k}\left(1-\tau_{k}-\beta_{k}\right)\right)\left(f\left(x_{k}\right)-x_{k}\right)+\sum_{k=1}^{n} \gamma_{k} \beta_{k}\left(f\left(z_{k}\right)-z_{k}\right) \\
& +\sum_{k=1}^{n} \alpha_{k}\left(f\left(y_{k}\right)-z_{k}\right) \\
= & x_{1}+\sum_{k=1}^{n} \mu_{k}\left(1-\gamma_{k}\left(1-\tau_{k}-\beta_{k}\right)\right) p_{k}+\sum_{k=1}^{n} \gamma_{k} \beta_{k} q_{k}+\sum_{k=1}^{n} \alpha_{k} r_{k} .
\end{aligned}
$$

It is easy to see that $\sum_{k=1}^{\infty} \mu_{k}\left(1-\gamma_{k}\left(1-\tau_{k}-\beta_{k}\right)\right) p_{k}<\infty$ since $\lim _{k \rightarrow \infty} p_{k} \neq 0$ and $\sum_{k=1}^{\infty} \mu_{k}<$ $\infty$. Similarly, we have $\sum_{k=1}^{\infty} \gamma_{k} \beta_{k} q_{k}<\infty$ since $\lim _{k \rightarrow \infty} q_{k} \neq 0$ and $\sum_{k=1}^{\infty} \beta_{k}<\infty$. This shows that $\left\{x_{n}\right\}$ is a divergent sequence since $\lim _{k \rightarrow \infty} r_{k} \neq 0$ and $\sum_{k=1}^{\infty} \alpha_{k}=\infty$. This contradicts the convergence of $\left\{x_{n}\right\}$. Hence $f(a)=a$ and $a$ is a fixed point of $f$.

Lemma 2.2 Let $C$ be a closed interval on the real line (can be unbounded) and let $f: C \rightarrow C$ be a continuous function. Let $\left\{\alpha_{n}\right\},\left\{\beta_{n}\right\},\left\{\mu_{n}\right\},\left\{\gamma_{n}\right\}$ and $\left\{\tau_{n}\right\}$ be sequences in $[0,1]$ with $0 \leq \tau_{n}+\beta_{n} \leq 1$ and $0 \leq \gamma_{n}+\alpha_{n} \leq 1$. Let $\left\{x_{n}\right\}$ be a sequence generated iteratively by $x_{1} \in C$ and

$$
\begin{aligned}
& z_{n}=\left(1-\mu_{n}\right) x_{n}+\mu_{n} f\left(x_{n}\right), \\
& y_{n}=\left(1-\tau_{n}-\beta_{n}\right) x_{n}+\tau_{n} z_{n}+\beta_{n} f\left(z_{n}\right), \\
& x_{n+1}=\left(1-\gamma_{n}-\alpha_{n}\right) z_{n}+\gamma_{n} y_{n}+\alpha_{n} f\left(y_{n}\right), \quad n \geq 1,
\end{aligned}
$$

where $\sum_{n=1}^{\infty} \alpha_{n}=\infty, \lim _{n \rightarrow \infty} \alpha_{n}=0, \sum_{n=1}^{\infty} \beta_{n}<\infty$ and $\sum_{n=1}^{\infty} \mu_{n}<\infty$.

If $\left\{x_{n}\right\}$ is bounded, then $\left\{x_{n}\right\}$ is convergent.

Proof Suppose $\left\{x_{n}\right\}$ is not convergent. Let $a=\liminf _{n} x_{n}$ and $b=\lim \sup _{n} x_{n}$. Then $a<b$. We first show that if $a<m<b$, then $f(m)=m$. Suppose $f(m) \neq m$. Without loss of generality, we suppose $f(m)-m>0$. Since $f$ is continuous, there exists $\delta$ with $0<\delta<b-a$ such that for $|x-m| \leq \delta$,

$$
f(x)-x>0
$$


Since $\left\{x_{n}\right\}$ is bounded and $f$ is continuous, $\left\{f\left(x_{n}\right)\right\}$ is bounded. Hence $\left\{z_{n}\right\},\left\{y_{n}\right\},\left\{f\left(z_{n}\right)\right\}$ and $\left\{f\left(y_{n}\right)\right\}$ are all bounded. Using

$$
\begin{aligned}
& x_{n+1}-x_{n}=\left(1-\gamma_{n}-\alpha_{n}\right)\left(z_{n}-x_{n}\right)+\gamma_{n}\left(y_{n}-x_{n}\right)+\alpha_{n}\left(f\left(y_{n}\right)-x_{n}\right), \\
& y_{n}-x_{n}=\tau_{n}\left(z_{n}-x_{n}\right)+\beta_{n}\left(f\left(z_{n}\right)-x_{n}\right), \\
& z_{n}-x_{n}=\mu_{n}\left(f\left(x_{n}\right)-x_{n}\right),
\end{aligned}
$$

we can easily show that $\left|z_{n}-x_{n}\right| \rightarrow 0,\left|y_{n}-x_{n}\right| \rightarrow 0$ and $\left|x_{n+1}-x_{n}\right| \rightarrow 0$. Thus there exists a positive integer $N$ such that for all $n>N$,

$$
\left|x_{n+1}-x_{n}\right|<\frac{\delta}{2}, \quad\left|y_{n}-x_{n}\right|<\frac{\delta}{2}, \quad\left|z_{n}-x_{n}\right|<\frac{\delta}{2} .
$$

Since $b=\lim \sup _{n} x_{n}>m$, there exists $k_{1}>N$ such that $x_{n_{k_{1}}}>m$. Let $n_{k_{1}}=k$, then $x_{k}>m$. For $x_{k}$, there exist two cases as follows.

(i) $x_{k}>m+\frac{\delta}{2}$, then $x_{k+1}>x_{k}-\frac{\delta}{2} \geq m$ using (2.3). So, we have $x_{k+1}>m$.

(ii) $m<x_{k}<m+\frac{\delta}{2}$, then $m-\frac{\delta}{2}<y_{k}<m+\delta$ and $m-\frac{\delta}{2}<z_{k}<m+\delta$ by (2.3). So, we obtain $\left|x_{k}-m\right|<\frac{\delta}{2}<\delta,\left|y_{k}-m\right|<\delta,\left|z_{k}-m\right|<\delta$. Hence

$$
f\left(x_{k}\right)-x_{k}>0, \quad f\left(y_{k}\right)-y_{k}>0, \quad f\left(z_{k}\right)-z_{k}>0 .
$$

We observe that

$$
\begin{aligned}
y_{k}-z_{k} & =\left(1-\tau_{k}-\beta_{k}\right)\left(x_{k}-z_{k}\right)+\beta_{k}\left(f\left(z_{k}\right)-z_{k}\right) \\
& =\mu_{k}\left(1-\tau_{k}-\beta_{k}\right)\left(x_{k}-f\left(x_{k}\right)\right)+\beta_{k}\left(f\left(z_{k}\right)-z_{k}\right) .
\end{aligned}
$$

From (2.2), (2.4) and (2.5), we have

$$
\begin{aligned}
x_{k+1}= & x_{k}+\mu_{k}\left(1-\gamma_{k}\left(1-\tau_{k}-\beta_{k}\right)\right)\left(f\left(x_{k}\right)-x_{k}\right)+\gamma_{k} \beta_{k}\left(f\left(z_{k}\right)-z_{k}\right) \\
& +\alpha_{k}\left(f\left(y_{k}\right)-z_{k}\right) \\
= & x_{k}+\mu_{k}\left(1-\gamma_{k}\left(1-\tau_{k}-\beta_{k}\right)\right)\left(f\left(x_{k}\right)-x_{k}\right)+\gamma_{k} \beta_{k}\left(f\left(z_{k}\right)-z_{k}\right) \\
& +\alpha_{k}\left(f\left(y_{k}\right)-y_{k}\right)+\alpha_{k}\left(y_{k}-z_{k}\right) \\
= & x_{k}+\mu_{k}\left(1-\gamma_{k}\left(1-\tau_{k}-\beta_{k}\right)\right)\left(f\left(x_{k}\right)-x_{k}\right)+\gamma_{k} \beta_{k}\left(f\left(z_{k}\right)-z_{k}\right) \\
& +\alpha_{k}\left(f\left(y_{k}\right)-y_{k}\right)+\alpha_{k}\left(\mu_{k}\left(1-\tau_{k}-\beta_{k}\right)\left(x_{k}-f\left(x_{k}\right)\right)+\beta_{k}\left(f\left(z_{k}\right)-z_{k}\right)\right) \\
= & x_{k}+\mu_{k}\left(1-\left(\gamma_{k}+\alpha_{k}\right)\left(1-\tau_{k}-\beta_{k}\right)\right)\left(f\left(x_{k}\right)-x_{k}\right)+\alpha_{k}\left(f\left(y_{k}\right)-y_{k}\right) \\
& +\beta_{k}\left(\gamma_{k}+\alpha_{k}\right)\left(f\left(z_{k}\right)-z_{k}\right) \\
> & x_{k} .
\end{aligned}
$$

Thus $x_{k+1}>x_{k}>m$. From (i) and (ii), we have $x_{k+1}>m$. Similarly, we get that $x_{k+2}>m$, $x_{k+3}>m, \ldots$. Thus we have $x_{n}>m$ for all $n>k=n_{k_{1}}$. So, $a=\lim _{k \rightarrow \infty} x_{n_{k}} \geq m$, which is a contradiction with $a<m$. Thus $f(m)=m$.

We next consider the following two cases. 
(i) There exists $x_{M}$ such that $a<x_{M}<b$. Then $f\left(x_{M}\right)=x_{M}$. It follows that

$$
z_{M}=\left(1-\mu_{M}\right) x_{M}+\mu_{M} f\left(x_{M}\right)=x_{M}
$$

and

$$
\begin{aligned}
y_{M} & =\left(1-\tau_{M}-\beta_{M}\right) x_{M}+\tau_{M} z_{M}+\beta_{M} f\left(z_{M}\right) \\
& =\left(1-\tau_{M}-\beta_{M}\right) x_{M}+\tau_{M} x_{M}+\beta_{M} f\left(x_{M}\right) \\
& =x_{M} .
\end{aligned}
$$

Hence, we obtain

$$
\begin{aligned}
x_{M+1} & =\left(1-\gamma_{M}-\alpha_{M}\right) z_{M}+\gamma_{M} y_{M}+\alpha_{M} f\left(y_{M}\right) \\
& =\left(1-\gamma_{M}-\alpha_{M}\right) x_{M}+\gamma_{M} x_{M}+\alpha_{M} f\left(x_{M}\right) \\
& =x_{M} .
\end{aligned}
$$

Similarly, we obtain $x_{M}=x_{M+1}=x_{M+2}=\cdots$. So, we conclude that $x_{n} \rightarrow x_{M}$. Since there exists $x_{n_{k}} \rightarrow a, x_{M}=a$. This shows that $x_{n} \rightarrow a$, which is a contradiction.

(ii) For all $n, x_{n} \leq a$ or $x_{n} \geq b$. Since $b-a>0$ and $\lim _{n \rightarrow \infty}\left|x_{n+1}-x_{n}\right|=0$, there exists $\bar{N}$ such that $\left|x_{n+1}-x_{n}\right|<\frac{(b-a)}{2}$ for $n>\bar{N}$. So, it is always that $x_{n} \leq a$ for $n>\bar{N}$; or it is always that $x_{n} \geq b$ for $n>\bar{N}$. If $x_{n} \leq a$ for $n>\bar{N}$, then $b=\lim _{l \rightarrow \infty} x_{n_{l}} \leq a$, which is a contradiction with $a<b$. If $x_{n} \geq b$ for $n>\bar{N}$, then $a=\lim _{k \rightarrow \infty} x_{n_{k}} \geq b$, which is a contradiction with $a<b$. Thus we conclude that $x_{n} \rightarrow a$. This completes the proof.

We are now ready to prove the main results of this paper.

Theorem 2.3 Let $C$ be a closed interval on the real line (can be unbounded) and let $f$ : $C \rightarrow C$ be a continuous function. Let $\left\{\alpha_{n}\right\},\left\{\beta_{n}\right\},\left\{\mu_{n}\right\},\left\{\gamma_{n}\right\}$ and $\left\{\tau_{n}\right\}$ be sequences in $[0,1]$ with $0 \leq \tau_{n}+\beta_{n} \leq 1$ and $0 \leq \gamma_{n}+\alpha_{n} \leq 1$. Let $\left\{x_{n}\right\}$ be a sequence generated iteratively by $x_{1} \in C$ and

$$
\begin{aligned}
& z_{n}=\left(1-\mu_{n}\right) x_{n}+\mu_{n} f\left(x_{n}\right), \\
& y_{n}=\left(1-\tau_{n}-\beta_{n}\right) x_{n}+\tau_{n} z_{n}+\beta_{n} f\left(z_{n}\right), \\
& x_{n+1}=\left(1-\gamma_{n}-\alpha_{n}\right) z_{n}+\gamma_{n} y_{n}+\alpha_{n} f\left(y_{n}\right), \quad n \geq 1,
\end{aligned}
$$

where $\sum_{n=1}^{\infty} \alpha_{n}=\infty, \lim _{n \rightarrow \infty} \alpha_{n}=0, \sum_{n=1}^{\infty} \beta_{n}<\infty$ and $\sum_{n=1}^{\infty} \mu_{n}<\infty$. If $\left\{x_{n}\right\}$ is bounded, then $\left\{x_{n}\right\}$ converges to a fixed point off.

Proof Let $\left\{x_{n}\right\}$ be a bounded sequence. Then, by Lemma (2.2), $\left\{x_{n}\right\}$ is a convergent sequence. Hence, by Lemma (2.1), it converges to a fixed point of $f$.

As a direct consequence of Theorem 2.3, we obtain the following result.

Theorem 2.4 Let $C$ be a closed interval on the real line (can be unbounded) and let $f$ : $C \rightarrow C$ be a continuous function. Let $\left\{\alpha_{n}\right\},\left\{\beta_{n}\right\},\left\{\mu_{n}\right\},\left\{\gamma_{n}\right\}$ and $\left\{\tau_{n}\right\}$ be sequences in $[0,1]$ 
with $0 \leq \tau_{n}+\beta_{n} \leq 1$ and $0 \leq \gamma_{n}+\alpha_{n} \leq 1$. Let $\left\{x_{n}\right\}$ be a sequence generated iteratively by $x_{1} \in C$ and

$$
\begin{aligned}
& z_{n}=\left(1-\mu_{n}\right) x_{n}+\mu_{n} f\left(x_{n}\right), \\
& y_{n}=\left(1-\tau_{n}-\beta_{n}\right) x_{n}+\tau_{n} z_{n}+\beta_{n} f\left(z_{n}\right), \\
& x_{n+1}=\left(1-\gamma_{n}-\alpha_{n}\right) z_{n}+\gamma_{n} y_{n}+\alpha_{n} f\left(y_{n}\right), \quad n \geq 1,
\end{aligned}
$$

where $\sum_{n=1}^{\infty} \alpha_{n}=\infty, \lim _{n \rightarrow \infty} \alpha_{n}=0, \sum_{n=1}^{\infty} \beta_{n}<\infty$ and $\sum_{n=1}^{\infty} \mu_{n}<\infty$.

Then $\left\{x_{n}\right\}$ converges to a fixed point off if and only if $\left\{x_{n}\right\}$ is bounded.

Corollary 2.5 Let $f:[a, b] \rightarrow[a, b]$ be a continuous function. Let $\left\{\alpha_{n}\right\},\left\{\beta_{n}\right\},\left\{\mu_{n}\right\},\left\{\gamma_{n}\right\}$ and $\left\{\tau_{n}\right\}$ be sequences in $[0,1]$ with $0 \leq \tau_{n}+\beta_{n} \leq 1$ and $0 \leq \gamma_{n}+\alpha_{n} \leq 1$. Let $\left\{x_{n}\right\}$ be a sequence generated iteratively by $x_{1} \in[a, b]$ and

$$
\begin{aligned}
& z_{n}=\left(1-\mu_{n}\right) x_{n}+\mu_{n} f\left(x_{n}\right), \\
& y_{n}=\left(1-\tau_{n}-\beta_{n}\right) x_{n}+\tau_{n} z_{n}+\beta_{n} f\left(z_{n}\right), \\
& x_{n+1}=\left(1-\gamma_{n}-\alpha_{n}\right) z_{n}+\gamma_{n} y_{n}+\alpha_{n} f\left(y_{n}\right), \quad n \geq 1,
\end{aligned}
$$

where $\sum_{n=1}^{\infty} \alpha_{n}=\infty, \lim _{n \rightarrow \infty} \alpha_{n}=0, \sum_{n=1}^{\infty} \beta_{n}<\infty$ and $\sum_{n=1}^{\infty} \mu_{n}<\infty$.

Then $\left\{x_{n}\right\}$ converges to a fixed point off.

If we take $\tau_{n}=\gamma_{n}=0$, then we obtain the following result.

Corollary 2.6 Let $C$ be a closed interval on the real line (can be unbounded) and let $f$ : $C \rightarrow C$ be a continuous function. Let $\left\{\alpha_{n}\right\},\left\{\beta_{n}\right\}$ and $\left\{\mu_{n}\right\}$ be sequences in $[0,1]$. Let $\left\{x_{n}\right\}$ be a sequence generated iteratively by $x_{1} \in C$ and

$$
\begin{aligned}
& z_{n}=\left(1-\mu_{n}\right) x_{n}+\mu_{n} f\left(x_{n}\right), \\
& y_{n}=\left(1-\beta_{n}\right) x_{n}+\beta_{n} f\left(z_{n}\right), \\
& x_{n+1}=\left(1-\alpha_{n}\right) z_{n}+\alpha_{n} f\left(y_{n}\right), \quad n \geq 1,
\end{aligned}
$$

where $\sum_{n=1}^{\infty} \alpha_{n}=\infty, \lim _{n \rightarrow \infty} \alpha_{n}=0, \sum_{n=1}^{\infty} \beta_{n}<\infty$ and $\sum_{n=1}^{\infty} \mu_{n}<\infty$.

Then $\left\{x_{n}\right\}$ converges to a fixed point off if and only if $\left\{x_{n}\right\}$ is bounded.

If we take $\tau_{n}+\beta_{n}=1$ and $\gamma_{n}+\alpha_{n}=1$, then we obtain the following result.

Corollary 2.7 Let $C$ be a closed interval on the real line (can be unbounded) and let $f$ : $C \rightarrow C$ be a continuous function. Let $\left\{\alpha_{n}\right\},\left\{\beta_{n}\right\}$ and $\left\{\mu_{n}\right\}$ be sequences in $[0,1]$. Let $\left\{x_{n}\right\}$ be a sequence generated iteratively by $x_{1} \in C$ and

$$
\begin{aligned}
& z_{n}=\left(1-\mu_{n}\right) x_{n}+\mu_{n} f\left(x_{n}\right), \\
& y_{n}=\left(1-\beta_{n}\right) z_{n}+\beta_{n} f\left(z_{n}\right), \\
& x_{n+1}=\left(1-\alpha_{n}\right) y_{n}+\alpha_{n} f\left(y_{n}\right), \quad n \geq 1,
\end{aligned}
$$


where $\sum_{n=1}^{\infty} \alpha_{n}=\infty, \lim _{n \rightarrow \infty} \alpha_{n}=0, \sum_{n=1}^{\infty} \beta_{n}<\infty$ and $\sum_{n=1}^{\infty} \mu_{n}<\infty$.

Then $\left\{x_{n}\right\}$ converges to a fixed point off if and only if $\left\{x_{n}\right\}$ is bounded.

Remark 2.8 Corollary 2.7 extends the main result obtained in [8] from the modified Ishikawa iteration to the modified Noor iteration.

\section{Numerical examples}

In this section, we give numerical examples to demonstrate the convergence of the algorithm defined in this paper. For convenience, we call the iteration (2.1) the CP-iteration.

Example 3.1 Let $f:[1, \infty) \longrightarrow[1, \infty)$ be defined by $f(x)=\sqrt{0.9 \ln x+1}$. Then $f$ is a continuous function. Use the initial point $x_{1}=3$ and the control conditions $\alpha_{n}=\frac{1}{(n+1)^{0.5}}$, $\beta_{n}=\frac{1}{(n+1)^{2.5}}, \mu_{n}=\frac{1}{(n+1)^{1.5}}, \tau_{n}=\frac{1}{n+1}$ and $\gamma_{n}=\frac{1}{7}$.

Table 1 Comparison of the convergence rate of Mann, Ishikawa, Noor and CP iterations for the function given in Example 3.1

\begin{tabular}{rlllll}
\hline $\boldsymbol{n}$ & Mann & Ishikawa & Noor & \multicolumn{2}{l}{ CP-iteration } \\
\cline { 5 - 6 } & $\boldsymbol{x}_{\boldsymbol{n}}$ & $\boldsymbol{x}_{\boldsymbol{n}}$ & $\boldsymbol{x}_{\boldsymbol{n}}$ & $\boldsymbol{x}_{\boldsymbol{n}}$ & $\left|\boldsymbol{f}\left(\boldsymbol{x}_{\boldsymbol{n}}\right)-\boldsymbol{x}_{\boldsymbol{n}}\right|$ \\
\hline 1 & 3.000000 & 3.000000 & 3.000000 & 3.000000 & 1.589769 \\
10 & 1.074110 & 1.071538 & 1.071437 & 1.043308 & 0.024408 \\
20 & 1.015478 & 1.014946 & 1.014925 & 1.008428 & 0.004658 \\
30 & 1.004821 & 1.004656 & 1.004650 & 1.002531 & 0.001394 \\
40 & 1.001822 & 1.001760 & 1.001757 & 1.000934 & 0.000514 \\
50 & 1.000777 & 1.000750 & 1.000749 & 1.000392 & 0.000216 \\
60 & 1.000360 & 1.000348 & 1.000348 & 1.000180 & 0.000099 \\
70 & 1.000178 & 1.000172 & 1.000172 & 1.000088 & 0.000048 \\
80 & 1.000093 & 1.000089 & 1.000089 & 1.000045 & 0.000025 \\
90 & 1.000050 & 1.000048 & 1.000048 & 1.000024 & 0.000013 \\
100 & 1.000028 & 1.000027 & 1.000027 & 1.000014 & 0.000007 \\
\hline
\end{tabular}

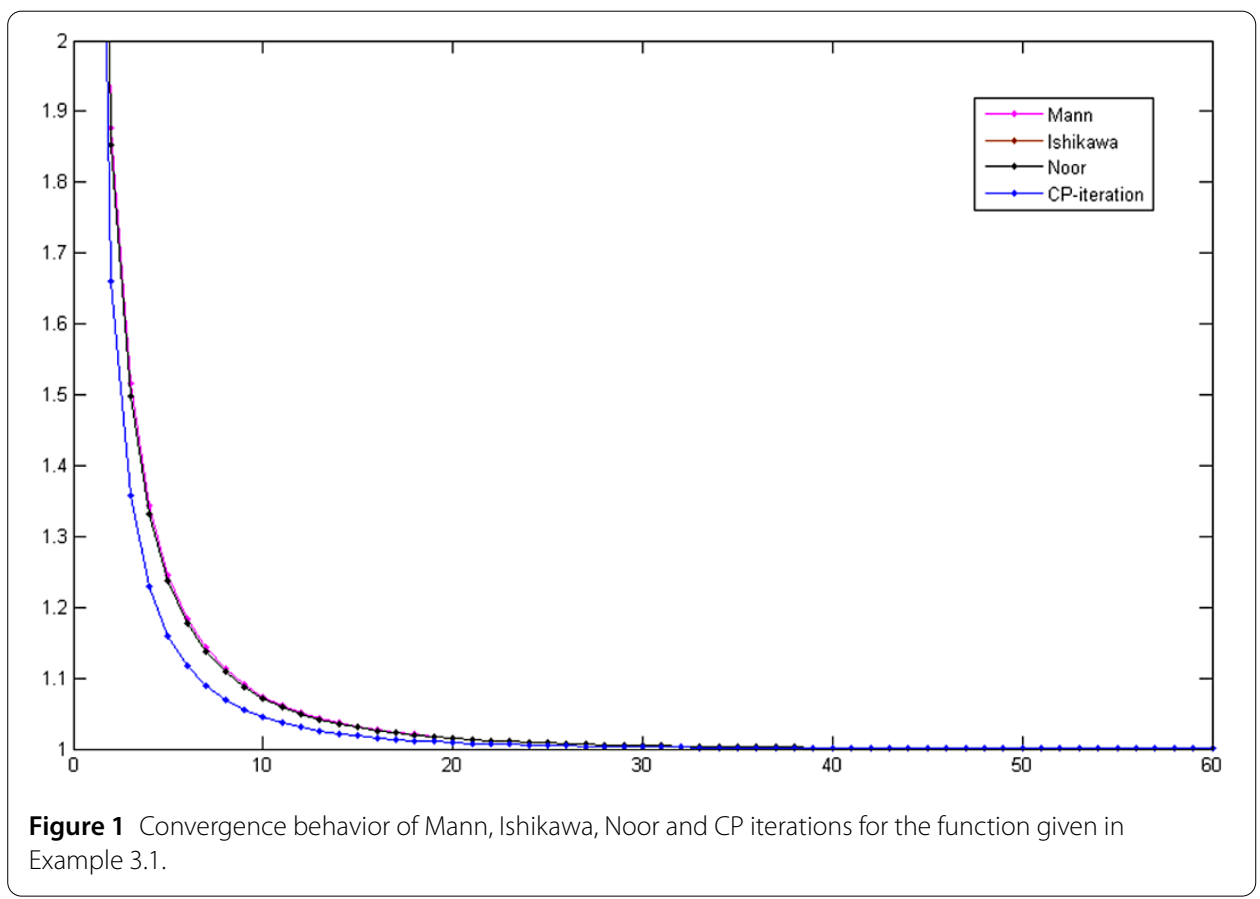


Table 2 Comparison of the convergence rate of Mann, Ishikawa, Noor and CP iterations for the function given in Example 3.2

\begin{tabular}{rlllll}
\hline $\boldsymbol{n}$ & Mann & Ishikawa & Noor & \multicolumn{2}{l}{ CP-iteration } \\
\cline { 5 - 6 } & $\boldsymbol{x}_{\boldsymbol{n}}$ & $\boldsymbol{x}_{\boldsymbol{n}}$ & $\boldsymbol{x}_{\boldsymbol{n}}$ & $\boldsymbol{x}_{\boldsymbol{n}}$ & $\left|\boldsymbol{f}\left(\boldsymbol{x}_{\boldsymbol{n}}\right)-\boldsymbol{x}_{\boldsymbol{n}}\right|$ \\
\hline 1 & 5.000000 & 5.000000 & 5.000000 & 5.000000 & 2.363932 \\
10 & 1.529828 & 1.504824 & 1.501931 & 1.359649 & 0.073668 \\
20 & 1.293319 & 1.284893 & 1.283943 & 1.224654 & 0.023218 \\
30 & 1.223891 & 1.219794 & 1.219335 & 1.187049 & 0.011033 \\
40 & 1.193599 & 1.191253 & 1.190991 & 1.171120 & 0.006204 \\
50 & 1.177752 & 1.176279 & 1.176115 & 1.162994 & 0.003827 \\
60 & 1.168542 & 1.167561 & 1.167452 & 1.158380 & 0.002505 \\
70 & 1.162807 & 1.162126 & 1.162050 & 1.155568 & 0.001710 \\
80 & 1.159055 & 1.158568 & 1.158513 & 1.153766 & 0.001205 \\
90 & 1.156509 & 1.156152 & 1.156112 & 1.152565 & 0.000870 \\
100 & 1.154730 & 1.154463 & 1.154433 & 1.151740 & 0.000641 \\
110 & 1.153458 & 1.153255 & 1.153233 & 1.151160 & 0.000480 \\
120 & 1.152530 & 1.152374 & 1.152357 & 1.150743 & 0.000365 \\
130 & 1.151842 & 1.151721 & 1.151707 & 1.150438 & 0.000281 \\
140 & 1.151325 & 1.151230 & 1.151219 & 1.150212 & 0.000218 \\
150 & 1.150931 & 1.150856 & 1.150847 & 1.150042 & 0.000171 \\
160 & 1.150628 & 1.150568 & 1.150561 & 1.149912 & 0.000136 \\
170 & 1.150393 & 1.150345 & 1.150339 & 1.149813 & 0.000108 \\
180 & 1.150209 & 1.150169 & 1.150165 & 1.149735 & 0.000087 \\
190 & 1.150063 & 1.150031 & 1.150027 & 1.149675 & 0.000070 \\
200 & 1.149947 & 1.149920 & 1.149918 & 1.149627 & 0.000057 \\
\hline
\end{tabular}

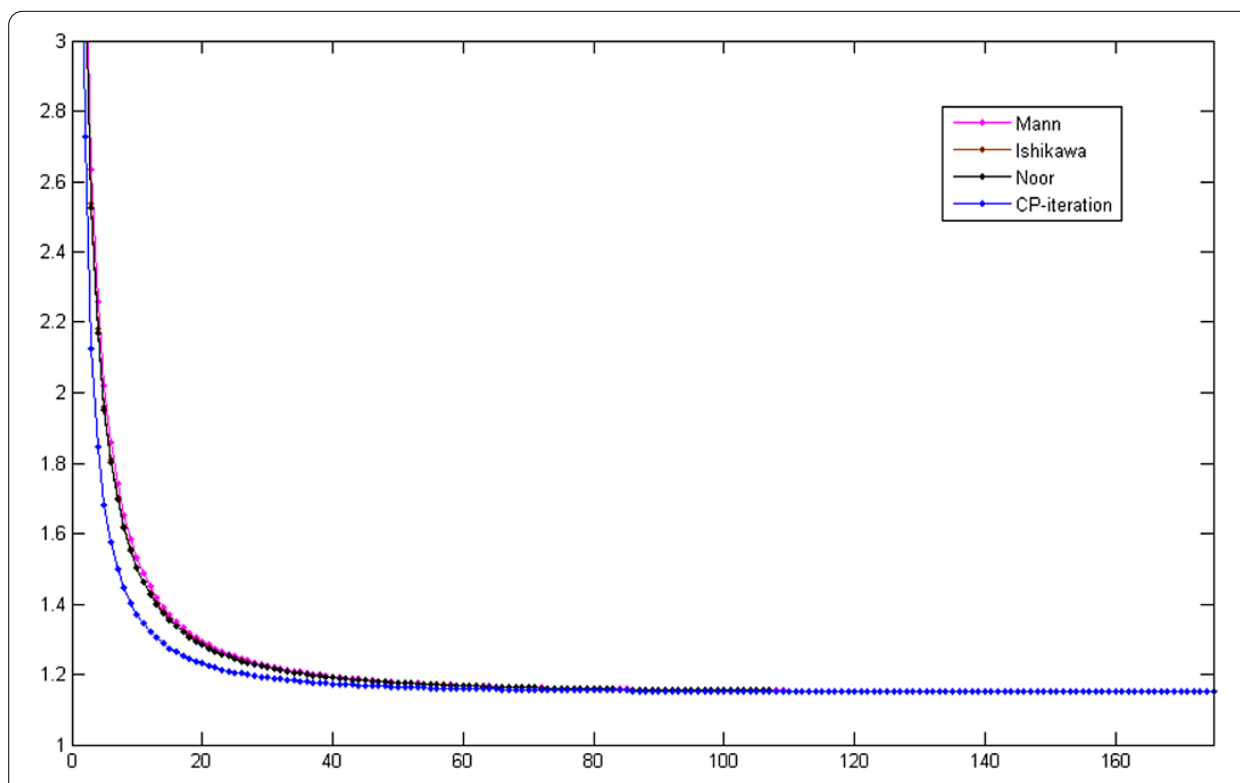

Figure 2 Convergence behavior of Mann, Ishikawa, Noor and CP iterations for the function given in Example 3.2.

Example 3.2 Let $f:[1, \infty) \longrightarrow[1, \infty)$ be defined by $f(x)=0.2 \sqrt{x-1}+\sqrt{x}$. Then $f$ is a continuous function. Use the initial point $x_{1}=5$ and the control conditions $\alpha_{n}=\frac{1}{(n+1)^{0.5}}$, $\beta_{n}=\frac{1}{(n+1)^{2}}, \mu_{n}=\frac{1}{(n+1)^{1.1}}, \tau_{n}=\frac{1}{n+1}$ and $\gamma_{n}=\frac{1}{5}$.

Remark 3.3 From Table 1, Figure 1, Table 2 and Figure 2, we observe that the sequence generated by the CP-iteration converges to a fixed point faster than that of Mann, Ishikawa and Noor iterations. 


\section{Competing interests}

The authors declare that they have no competing interests.

\section{Authors' contributions}

PC and NP contributed equally. All authors read and approved the final manuscript.

\section{Acknowledgements}

The authors wish to thank Professor Suthep Suantai for the valuable guidance and suggestion. The first author was supported by the Thailand Research Fund, the Commission on Higher Education, and University of Phayao under Grant No. MRG5580016.

Received: 22 November 2012 Accepted: 15 April 2013 Published: 29 April 2013

\section{References}

1. Mann, WR: Mean value methods in iteration. Proc. Am. Math. Soc. 4, 506-510 (1953)

2. Borwein, D, Borwein, J: Fixed point iterations for real functions. J. Math. Anal. Appl. 157, 112-126 (1991)

3. Ishikawa, S: Fixed points by a new iteration method. Proc. Am. Math. Soc. 44, 147-150 (1974)

4. Qing, Y, Qihou, L: The necessary and sufficient condition for the convergence of Ishikawa iteration on an arbitrary interval. J. Math. Anal. Appl. 323, 1383-1386 (2006)

5. Qing, Y, Cho, SY, Qin, X: Convergence of Ishikawa iteration with error terms on an arbitrary interval. Commun. Korean Math. Soc. 26, 229-235 (2011)

6. Noor, MA: New approximation schemes for general variational inequalities. J. Math. Anal. Appl. 251, $217-229$ (2000)

7. Phuengrattana, W, Suantai, S: On the rate of convergence of Mann Ishikawa, Noor and SP iterations for continuous functions on an arbitrary interval. J. Comput. Appl. Math. 235, 3006-3014 (2011)

8. Suwana-adth, $W$ : The necessary and sufficient condition for the convergence of a new fixed point approximation method for continuous functions on an arbitrary interval. Thai J. Math. 8, 627-632 (2010)

doi:10.1186/1029-242X-2013-214

Cite this article as: Cholamjiak and Pholasa: Approximating fixed points for continuous functions on an arbitrary

interval. Journal of Inequalities and Applications 2013 2013:214.

\section{Submit your manuscript to a SpringerOpen ${ }^{\circ}$ journal and benefit from:}

- Convenient online submission

- Rigorous peer review

- Immediate publication on acceptance

- Open access: articles freely available online

- High visibility within the field

- Retaining the copyright to your article 